\title{
PRODUÇÃO DE MUDAS DE CANAFÍSTULA CULTIVADAS EM LATOSSOLO VERMELHO AMARELO ÁLICO EM RESPOSTA A MACRONUTRIENTES
}

\author{
Cezar Augusto Fonseca e Cruz¹, Haroldo Nogueira de Paiva², Ana Catarina Monteiro Carvalho Mori da Cunha³, \\ Júlio César Lima Neves ${ }^{4}$
}

(recebido: 4 de agosto de 2009; aceito: 28 de outubro de 2011)

\begin{abstract}
RESUMO: Peltophorum dubium (canafístula) é uma espécie de rápido crescimento, rusticidade e ótima para uso em reflorestamentos mistos de áreas degradadas. Neste trabalho, objetivou-se avaliar o efeito de doses de N, P, K, Ca, Mg e S sobre crescimento e índices de qualidade de mudas e determinar a dose recomendada dos mesmos para estabelecer um adequado programa de adubação. No trabalho, conduzido em casa de vegetação, foi utilizado Latossolo Vermelho Amarelo álico como substrato. Delimitou-se o experimento por meio de matriz baconiana, avaliando-se três doses dos seis macronutrientes e dois tratamentos adicionais, um com doses de referência e outro sem adição de nutrientes. Adotou-se o delineamento inteiramente casualizado com quatro repetições. Verificou-se, por meio de análise dos contrastes ortogonais, resposta significativa de todas as variáveis estudadas à aplicação dos macronutrientes, exceto para a relação H/D. Os nutrientes que mais surtiram efeitos significativos foram o P e o N, sendo recomendada dose de 540 mg/ $\mathrm{dm}^{3}$ de $\mathrm{P}$ e $50 \mathrm{mg} / \mathrm{dm}^{3}$ de $\mathrm{N}$. Não foi verificada resposta à aplicação de $\mathrm{K}, \mathrm{Ca}, \mathrm{Mg}$ e $\mathrm{S}$, para a maioria das características avaliadas, o que indica que a espécie tem baixo requerimento por esses nutrientes.
\end{abstract}

Palavras-chave: Nutrição de plantas, características morfológicas, Peltophorum dubium.

\section{PRODUCTION OF CANAFÍSTULA SEEDLINGS CULTIVATED IN RED-YELLOW ALIC LATOSOL IN RESPONSE TO MACRONUTRIENTS}

\begin{abstract}
Peltophorum dubium (canafístula) is a fast-growing, rustic species excellent for use in combined reforestation of degraded areas. The objective of this study was to evaluate the effect of doses of $N, P, K, C a, M g$ and $S$ on seedling growth and quality indices; and to determine the recommended dose of these elements for establishment of a suitable fertilization program. The study was conducted in a greenhouse using red-yellow alic latosol as substrate. The experiment was designed following a Baconian matrix, evaluating three doses of the six macronutrients and two additional treatments, one with reference doses and another with no addition of nutrients. A completely randomized design was adopted with four replicates. Analysis using orthogonal contrasts revealed a significant response from all variables being studied to application of macronutrients, except the H/D ratio. The nutrients $P$ and $N$ had the most significant effects, $540 \mathrm{mg} / \mathrm{dm} 3$ being the recommended dose of $P$, and $50 \mathrm{mg} / \mathrm{dm} 3$ being the recommended dose of $\mathrm{N}$. No response was observed to application of $\mathrm{K}, \mathrm{Ca}, \mathrm{Mg}$ and $\mathrm{S}$, for most traits being assessed, indicating that the species has a low requirement for these nutrients.
\end{abstract}

Key words: Plant nutrition, morphological traits, Peltophorum dubium.

\section{INTRODUÇÃO}

Da família Caesalpinaceae, a espécie Peltophorum dubium (Spreng.) Taub. (canafístula, angico-cangalha, angico-amarelo, faveiro, tamboril) é uma árvore nativa, frequente em todo o domínio da floresta estacional semidecidual; desempenha papel pioneiro em áreas abertas, capoeiras e matas degradadas (CARVALHO, 2003; MACHIORI, 1997). Carvalho (2003) classifica a espécie como pertencente, no estudo de sucessão, ao grupo das secundárias iniciais. A canafístula é medianamente tolerante ao frio, pode ser usada para a arborização urbana, pois a árvore, além de muito ornamental quando em florescimento, proporciona ótima sombra quando isolada e cercas divisórias de diversas propriedades (LORENZI, 1992; VENTURIN et al., 1999). Pelo seu rápido crescimento e rusticidade, é comumente encontrada colonizando pastagens, ocupando clareiras e bordas de matas, sendo também utilizada para a composição de reflorestamentos mistos de áreas degradadas de preservação permanente (LORENZI, 1992).

\footnotetext{
${ }^{1}$ Engenheiro Florestal, M.Sc. em Ciências Florestais, Analista do Instituto Estadual de Florestas - Av. Doutor David Benedito Ottoni, 257, Jardim dos Estados - 37701-069 - Poços de Caldas, MG - ceaufoc1@yahoo.com.br

${ }^{2}$ Engenheiro Florestal, Professor D.Sc. em Agronomia - Departamento de Engenharia Florestal - Centro de Ciências Agrárias - Universidade Federal de Viçosa - 36570-000 - Viçosa, MG - hnpaiva@ufv.br

${ }^{3}$ Engenheira Florestal, Professora D.Sc. em Ciência Florestal - Instituo Federal de Educação, Ciência e Tecnologia do Sudeste de Minas Gerais Av. Dr. José Sebastião da Paixão, S/N, Lindo Vale - 36180-000 - Rio Pomba, MG - catarina_mori@yahoo.com.br

${ }^{4}$ Engenheiro Agrônomo, Professor D.Sc. em Produção Vegetal - Departamento de Solos e Nutrição de Plantas - Centro de Ciências Agrárias Universidade Federal de Viçosa - 36570-000 - Viçosa, MG - julio_n2003@yahoo.com.br
}

Cerne, Lavras, v. 18, n. 1, p. 87-98, jan./mar. 2012 
A degradação de pastagens é um problema comum em diversas propriedades rurais de Minas Gerais, sendo causada pelo manejo inadequado das mesmas (CARVALHO, 1997). As consequências do manejo incorreto são a perda do solo por erosão e a baixa retenção de água da chuva, problemas estes que se agravam em regiões de topografia acidentada como a Zona da Mata mineira e a região do Rio Doce.

As árvores podem ser usadas para minimizar os efeitos prejudiciais da erosão e da degradação de pastagens, tendo em vista a potencialidade que as espécies florestais apresentam no controle da erosão, por aumentar a capacidade de infiltração de água e por promover melhorias nas características físicas e químicas do solo. Dentre as espécies que podem ser usadas na recuperação dessas áreas devem ser escolhidas aquelas adaptadas às características edafoclimáticas da região, preferencialmente as leguminosas, pela sua capacidade de se associar a microorganismos fixadores de nitrogênio.

No entanto, o sucesso na utilização de espécies florestais nativas, principalmente em projetos de recuperação de áreas degradadas, depende do conhecimento dos seus requerimentos nutricionais, visando a aperfeiçoar o sistema de produção de mudas e, consequentemente, aumentar o seu potencial de sobrevivência e crescimento após o plantio no campo.

O conhecimento dos requerimentos nutricionais e da capacidade de adaptação das espécies florestais nativas a ambientes distintos ainda é incipiente. De acordo com Gonçalves et al. (1992), respostas positivas a diferentes nutrientes têm sido observadas quando se realiza a adubação de mudas florestais, bem como variações na concentração, absorção e eficiência de uso de nutrientes entre espécies pioneiras, secundárias e clímaxes, tanto na fase de viveiro como no campo.

O substrato usado na produção de mudas de espécies florestais nativas é comumente proveniente de solos e subsolos pobres em nutrientes, o que torna indispensável a utilização de fertilizantes, de modo a atender às exigências nutricionais das espécies o que, segundo Carneiro (1995), favorece a sobrevivência e o crescimento inicial das mudas após o plantio no campo.

Para o aproveitamento do potencial da canafístula, na recuperação de áreas degradadas faz-se necessário o desenvolvimento de estudos que visem ao estabelecimento de critérios para a recomendação de fertilização, e a determinação das doses recomendadas dos nutrientes. A determinação dessas doses e o relacionamento das mesmas com as respostas da planta são consideradas como alternativa para otimizar a recomendação das quantidades de nutrientes a serem aplicadas ao substrato usado na produção das mudas.

Diante do exposto, os objetivos desse trabalho foram: avaliar o efeito da aplicação de doses dos macronutrientes no solo sobre o crescimento e qualidade das mudas de canafístula cultivadas em Latossolo Vermelho Amarelo álico e; determinar a dose recomendada dos mesmos, a fim de estabelecer um adequado programa de adubação.

\section{MATERIAL E MÉTODOS}

O estudo foi conduzido no município de Viçosa, MG, no período de janeiro a junho de 2006. Situado nas coordenadas $20^{\circ} 45^{\prime} \mathrm{S}$ e $42^{\circ} 55^{\prime} \mathrm{W}$, na Zona da Mata de Minas Gerais, o município possui clima classificado como tropical de altitude, com verões chuvosos e invernos frios e secos, do tipo Cwb, pelo sistema de Köppen, com precipitação média anual de $1.221 \mathrm{~mm}$.

Para a realização do estudo, foi usado um Latossolo Vermelho Amarelo álico (argila $55 \%$; silte $8 \%$; areia grossa $21 \%$; areia fina $16 \%$ ) retirado da camada abaixo de $20 \mathrm{~cm}$ de profundidade, seco ao ar e peneirado em malha de 4 mm de diâmetro, cuja caracterização química está apresentada na Tabela 1.

Tabela 1 - Análise química do solo utilizado na produção das mudas antes da aplicação dos tratamentos.

Table 1 - Chemical analysis of the soil used for seedling production before applying the treatments.

\begin{tabular}{|c|c|c|c|c|c|c|c|c|c|c|c|}
\hline \multirow{2}{*}{ Solo } & $\mathrm{pH}$ & $\mathrm{P}$ & K & $\mathrm{Ca}^{2+}$ & $\mathrm{Mg}^{2+}$ & $\mathrm{Al}^{3+}$ & $\mathrm{H}+\mathrm{Al}$ & SB & $(\mathrm{T})$ & $\mathrm{V}$ & $\mathrm{m}$ \\
\hline & $\mathrm{H}_{2} \mathrm{O}$ & \multicolumn{2}{|c|}{$\mathrm{mg} / \mathrm{dm}^{3}$} & \multicolumn{6}{|c|}{$\mathrm{cmol}_{\mathrm{c}} / \mathrm{dm}^{3}$} & \multicolumn{2}{|c|}{$\%$} \\
\hline LVA álico & 4,80 & 1,40 & 32 & 0,20 & 0,00 & 0,80 & 3,96 & 0,28 & 4,24 & 7 & 74 \\
\hline $\begin{array}{l}\text { pH em água } \\
\text { P e K - Extr } \\
\mathrm{CTC}(\mathrm{T})-\mathrm{C} \\
\mathrm{Ca}^{2+}, \mathrm{Mg}^{2+}\end{array}$ & $\begin{array}{l}\mathrm{Cl} \text { e C } \\
\text { r Mehl } \\
\text { acidad } \\
3^{3^{+}}-\mathrm{E}\end{array}$ & $\begin{array}{l}- \text { Rel } \\
1 \\
\text { troca } \\
\text { or KCl }\end{array}$ & $\begin{array}{l}: 2,5 \\
\text { ica } \\
1 / L\end{array}$ & & $\begin{array}{l}\mathrm{SB}- \\
\mathrm{V}-\mathrm{I} \\
\mathrm{MO} \\
\mathrm{m}-\end{array}$ & $\begin{array}{l}\text { ma de } \\
\text { ce de } \\
\text { Org x } \\
\text { Iração }\end{array}$ & $\begin{array}{l}\text { es trocá } \\
\text { Iração p } \\
724-\mathrm{M} \\
\text { alumín }\end{array}$ & $\begin{array}{l}\text { ases } \\
\text { lo Wa }\end{array}$ & -Black & & \\
\hline
\end{tabular}

Cerne, Lavras, v. 18, n. 1, p. 87-98, jan./mar. 2012 
O solo utilizado neste estudo foi proveniente de área próxima à Viçosa, sendo escolhido em razão da sua ocorrência predominante na região da Zona da Mata mineira.

Os tratamentos foram obtidos segundo matriz baconiana (TURRENT, 1979), na qual um dos nutrientes é fornecido em quantidades variáveis, enquanto os outros são mantidos em um nível referencial. Avaliaram-se seis nutrientes (nitrogênio, fósforo, potássio, cálcio, magnésio e enxofre), em três diferentes doses, e ainda dois tratamentos adicionais, sendo um com doses de referência e outro sem adição de nutrientes, totalizando 20 tratamentos que foram dispostos em delineamento inteiramente casualizado, com quatro repetições. Os tratamentos foram delimitados de maneira que, quando a quantidade de um nutriente estivesse variando, as doses dos demais estariam fixas, sendo que a variação das doses e dos nutrientes em cada tratamento pode ser visualizada na Tabela 2.

Tabela 2 - Tratamentos, obtidos pela matriz baconiana, com as doses dos nutrientes aplicadas no substrato para a produção das mudas.

Table 2 - Treatments, obtained by the Baconian matrix, with the doses of nutrients applied to the substrate for seedling production.

\begin{tabular}{lccc}
\hline Tratamentos & Unidades & Tratamentos & Unidades \\
\hline $\begin{array}{l}\text { DR } * \\
\text { Solo sem }\end{array}$ & $\mathrm{mg} / \mathrm{dm}^{3} / \mathrm{cmol}_{\mathrm{c}} / \mathrm{dm}^{3}$ & $\mathrm{~K}=200 * *$ & $\mathrm{mg} / \mathrm{dm}^{3}$ \\
correção & - & $\mathrm{Ca}=0,8 * *$ & $\mathrm{cmol}_{\mathrm{c}} / \mathrm{dm}^{3}$ \\
$\mathrm{~N}=50 * *$ & $\mathrm{mg} / \mathrm{dm}^{3}$ & $\mathrm{Ca}=1,2 * *$ & $\mathrm{cmol}_{\mathrm{c}} / \mathrm{dm}^{3}$ \\
$\mathrm{~N}=150 * *$ & $\mathrm{mg} / \mathrm{dm}^{3}$ & $\mathrm{Ca}=1,4 * *$ & $\mathrm{cmol}_{\mathrm{c}} / \mathrm{dm}^{3}$ \\
$\mathrm{~N}=200 * *$ & $\mathrm{mg} / \mathrm{dm}^{3}$ & $\mathrm{Mg}=0,2 * *$ & $\mathrm{cmol}_{\mathrm{c}} / \mathrm{dm}^{3}$ \\
$\mathrm{P}=150 * *$ & $\mathrm{mg} / \mathrm{dm}^{3}$ & $\mathrm{Mg}=0,6 * *$ & $\mathrm{cmol} / \mathrm{dm}^{3}$ \\
$\mathrm{P}=450 * *$ & $\mathrm{mg} / \mathrm{dm}^{3}$ & $\mathrm{Mg}=0,8 * *$ & $\mathrm{cmol} / \mathrm{dm}^{3}$ \\
$\mathrm{P}=600 * *$ & $\mathrm{mg} / \mathrm{dm}^{3}$ & $\mathrm{~S}=20 * *$ & $\mathrm{mg} / \mathrm{dm}^{3}$ \\
$\mathrm{~K}=50 * *$ & $\mathrm{mg} / \mathrm{dm}^{3}$ & $\mathrm{~S}=60 * *$ & $\mathrm{mg} / \mathrm{dm}^{3}$ \\
$\mathrm{~K}=150 * *$ & $\mathrm{mg} / \mathrm{dm}^{3}$ & $\mathrm{~S}=80 * *$ & $\mathrm{mg} / \mathrm{dm}^{3}$ \\
\hline
\end{tabular}

*Valores da dose de referência: $\mathrm{N}=100 \mathrm{mg} / \mathrm{dm}^{3} ; \mathrm{P}=300 \mathrm{mg} /$ $\mathrm{dm}^{3} ; \mathrm{K}=100 \mathrm{mg} / \mathrm{dm}^{3} ; \mathrm{Ca}=1 \mathrm{cmol} / \mathrm{dm}^{3} ; \mathrm{Mg}=0,4 \mathrm{cmol}_{\mathrm{c}} / \mathrm{dm}^{3}$; $\mathrm{S}=40 \mathrm{mg} / \mathrm{dm}^{3}$.

**Em cada tratamento apenas o nutriente indicado tem a dose variando, permanecendo as doses dos demais nutrientes iguais à dose de referência.

A unidade experimental foi constituída por um vaso de polietileno rígido com capacidade de $2,1 \mathrm{dm}^{3} \mathrm{de}$ solo e uma muda. A espécie selecionada para realização deste experimento foi a canafístula, cujas sementes foram originárias do Laboratório de Análise de Sementes (LASO) da Fundação Estadual de Pesquisa Agropecuária do Rio Grande do Sul (FEPAGRO).

Foram pesados 2,1 kg do solo usado no experimento e, em seguida, acondicionado em sacos plásticos. Posteriormente, foi adicionado ao solo doses de corretivos, sendo empregada um mistura de $\mathrm{CaCO}_{3}$ e $\mathrm{MgCO}_{3}$, nas quantidades definidas pelos tratamentos (Tabela 2) e homogeneizados. Depois da aplicação dos corretivos, seguiu-se um período de incubação de 30 dias, mantendo o teor de água próximo à capacidade de campo.

Decorridos os 30 dias, foram adicionados os demais nutrientes, de acordo com as quantidades definidas na Tabela 2. A aplicação de nitrogênio e potássio foi igualmente parcelada em 0, 30, 50, 70 e 90 dias após a semeadura.

Antes da semeadura aplicou-se, em todos os tratamentos, uma solução de micronutrientes composta por: boro (B) $\left(0,81 \mathrm{mg} / \mathrm{dm}^{3} \mathrm{de}_{3} \mathrm{BO}_{3}\right)$, cobre (Cu) $(1,33$ $\mathrm{mg} / \mathrm{dm}^{3}$ de $\left.\mathrm{CuSO}_{4} \cdot 5 \mathrm{H}_{2} \mathrm{O}\right)$, molibdênio (Mo) $\left(0,15 \mathrm{mg} / \mathrm{dm}^{3}\right.$ de $\left.\left(\mathrm{NH}_{4}\right)_{6} \mathrm{Mo}_{7} \mathrm{O}_{24} \cdot 4 \mathrm{H}_{2} \mathrm{O}\right)$, manganês $(\mathrm{Mn})\left(3,66 \mathrm{mg} / \mathrm{dm}^{3}\right.$ de $\mathrm{MnCl}_{2} \cdot \mathrm{H}_{2} \mathrm{O}$ e zinco $(\mathrm{Zn})\left(4,0 \mathrm{mg} / \mathrm{dm}^{3}\right.$ de $\left.\mathrm{ZnSO}_{4} \cdot 7 \mathrm{H}_{2} \mathrm{O}\right)$ como indicado por Alvarez (1974).

Fez-se a semeadura de 10 sementes diretamente nos vasos. O primeiro raleio foi realizado 15 dias após a emergência das plântulas, deixando-se duas plântulas por vaso, e o segundo aos 30 dias, permanecendo então apenas uma plântula por vaso. $\mathrm{O}$ teor de umidade do solo foi controlado pelo monitoramento diário, a fim de mantêlo próximo a $60 \%$ da capacidade de campo.

Aos 120 dias após a semeadura, realizou-se a medição da altura $(\mathrm{H})$, utilizando-se régua graduada em centímetros, e diâmetro do coleto (DC) das mudas com paquímetro com precisão de $0,01 \mathrm{~mm}$, quando, então, deuse por encerrado o experimento. Em seguida, as plantas foram colhidas e subdivididas em raiz e parte aérea, lavadas em água destilada e postas a secar em estufa com circulação forçada de ar a $60^{\circ} \mathrm{C}$ até que atingissem peso constante.

A determinação do peso de matéria seca de raiz (MSR) e peso de matéria seca da parte aérea (MSPA) foi realizada em balança analítica com precisão de 0,01 g, e o peso de matéria seca total (MST) foi obtido pela soma da MSR e da MSPA. Com esses dados, calcularam-se os índices de qualidade de mudas: altura de parte aérea por diâmetro do coleto (H/D), altura de parte aérea por peso de matéria seca de parte aérea (H/MSPA), peso de matéria

Cerne, Lavras, v. 18, n. 1, p. 87-98, jan./mar. 2012 
seca de parte aérea por peso de matéria seca de raiz (MSPA/ MSR), bem como o Índice de Qualidade de Dickson (IQD), de acordo com a fórmula (DICKSON et al., 1960):

$$
\mathrm{IQD}=\mathrm{MST} /(\mathrm{H} / \mathrm{D}+\mathrm{MSPA} / \mathrm{MSR})
$$

onde: MST - Peso de matéria seca total (g); H - Altura de parte aérea (cm); D - Diâmetro do coleto (mm); MSR Peso de matéria seca de raiz (g) e; MSPA - Peso de matéria seca da parte aérea (g).

Os dados foram interpretados estatisticamente por meio de contrastes ortogonais, análises de variância e regressão, utilizando-se o programa SAEG (Sistema de Análises Estatística e Genética) e o Statistica. Testou-se o efeito da adição de macronutrientes versus não adição por meio de um contraste entre as médias do tratamento sem adição de nutrientes (solo em correção) versus a média dos demais tratamentos. Para a obtenção das equações, utilizaram-se quatro pontos, sendo três referentes às doses testadas para cada nutriente e um referente ao tratamento adicional de referência, que foi utilizado em todos os nutrientes. As curvas de resposta à adição de doses de cada nutriente resultaram em modelos de regressão nos quais os coeficientes foram testados com base nos valores do quadrado médio do resíduo da ANOVA, sendo analisada a 1,5 e $10 \%$ de probabilidade. Adicionalmente, observou-se o $\mathrm{R}^{2}$, a significância dos betas da equação e o significado biológico dos modelos. Com essas equações, determinaram-se as doses recomendadas dos macronutrientes, para a obtenção de $90 \%$ dos valores máximos estimados das variáveis estudadas.

\section{RESULTADOS E DISCUSSÃO}

A análise de contrastes ortogonais entre os tratamentos com fertilizantes versus sem fertilizantes indicou que os tratamentos com adição de sais foram significativamente superiores em todas as características avaliadas, exceto para a relação H/D. Isso indica que a aplicação de fertilizantes formulados à base de macronutrientes possibilita aumento significativo do crescimento das mudas de canafístula, bem como proporciona mudas de melhor qualidade.

Observaram-se baixas médias de crescimento e qualidade de mudas em todas as características, exceto para as relações H/D e H/MSPA, no tratamento sem adição de sais, sendo que os valores médios verificados para as diversas características avaliadas, em geral, aumentaram em razão da aplicação de doses crescentes de macronutrientes, à exceção da relação H/MSPA (Tabela 3). Esse fato indica que os teores de nutrientes originalmente existentes no solo utilizado como substrato para a produção de mudas de canafístula são baixos. Essa resposta era esperada, tendo em vista que os nutrientes minerais desempenham diversas funções na planta e, consequentemente, a omissão de qualquer um deles acarreta diminuição no ritmo de crescimento, bem como pode ser prejudicial à qualidade das mudas produzidas.

Por ser de fácil medição e não ser um método destrutivo, a altura da parte área $(\mathrm{H})$ é amplamente utilizada para estimar o padrão de qualidade de mudas de espécies florestais nos viveiros (GOMES, 2001); ainda, é considerada uma característica importante para estimar o crescimento no campo (REIS et al., 1991).

Para todos os macronutrientes, as mudas referentes ao tratamento controle (sem aplicação de fertilizantes) apresentaram crescimento em $\mathrm{H}$ inferior, em média, de 5 a 6 vezes, comparativamente às maiores médias observadas para cada nutriente isoladamente (Tabela 3).

De acordo com Paiva e Gomes (2000), mudas de espécies arbóreas estão aptas ao plantio no campo quando a H estiver entre 15 e $30 \mathrm{~cm}$. Ao final desse experimento, para todos os tratamentos nos quais foram aplicadas doses crescentes de macronutrientes, verificou-se que os valores médios de $\mathrm{H}$ encontram-se acima dos valores supramencionados, podendo-se inferir, assim, que as mudas de canafístula, segundo esse critério, estariam aptas ao plantio no campo.

Segundo Souza et al. (2006), a avaliação do diâmetro do coleto (DC) é de fundamental importância na avaliação do potencial da muda para sobrevivência e crescimento após o plantio. Assim, as mudas devem apresentar DC maiores para exprimir melhor equilíbrio do crescimento da parte aérea (CARNEIRO, 1995), principalmente quando se exige maior endurecimento delas (GOMES, 2001). No entanto, a definição de um valor de DC que exprima com fidelidade o real padrão de qualidade das mudas para o plantio em local definido depende da espécie, do local, do método e das técnicas de produção (GOMES, 2001). Neste trabalho, observaramse valores de DC mínimo de 5,53 e máximo de 7,59 mm (Tabela 3), com maior parte dos valores observados entre 6,0 e 7,0 mm, sendo que a aplicação de doses crescentes de macronutrientes praticamente triplicou os valores de DC das mudas cultivadas em substrato fertilizado em comparação ao não fertilizado, para a maioria dos macronutrientes.

Cerne, Lavras, v. 18, n. 1, p. 87-98, jan./mar. 2012 
Tabela 3 - Médias das características ${ }^{1}$ avaliadas para canafístula em razão da aplicação de N, P, K, Ca, Mg e S, aos 120 dias após a semeadura, cultivadas em latossolo vermelho amarelo álico.

Table 3 - Means of traits ${ }^{1}$ evaluated in canafístula as a function of N, P, K, Ca, Mg and S application, 120 days after planting, using red-yellow alic latosol for cultivation.

\begin{tabular}{|c|c|c|c|c|c|c|c|c|c|c|}
\hline Nutriente & Dose & $\mathrm{H}(\mathrm{cm})$ & DC (mm) & MSPA (g) & MSR (g) & MST (g) & $\mathrm{H} / \mathrm{D}$ & H/MSPA & MSPA/MSR & IQD \\
\hline $\mathrm{N}$ & 0 & 4,90 & 2,04 & 0,19 & 0,39 & 0,57 & 2,46 & 26,22 & 0,51 & 0,20 \\
\hline $\mathrm{N}$ & 50 & 29,35 & 7,59 & 7,08 & 3,69 & 10,76 & 3,90 & 4,31 & 1,91 & 1,86 \\
\hline $\mathrm{N}$ & 100 & 26,83 & 7,15 & 6,01 & 3,26 & 9,27 & 3,77 & 4,45 & 1,85 & 1,67 \\
\hline $\mathrm{N}$ & 150 & 26,10 & 6,64 & 5,97 & 3,38 & 9,35 & 3,93 & 4,36 & 1,77 & 1,66 \\
\hline $\mathrm{N}$ & 200 & 26,19 & 6,39 & 5,46 & 3,06 & 8,52 & 4,10 & 4,86 & 1,79 & 1,47 \\
\hline $\mathrm{P}$ & 0 & 4,90 & 2,04 & 0,19 & 0,39 & 0,57 & 2,46 & 26,22 & 0,51 & 0,20 \\
\hline $\mathrm{P}$ & 150 & 20,35 & 5,53 & 3,33 & 2,14 & 5,46 & 3,68 & 6,42 & 1,57 & 1,09 \\
\hline $\mathrm{P}$ & 300 & 26,83 & 7,15 & 6,01 & 3,26 & 9,27 & 3,77 & 4,45 & 1,85 & 1,67 \\
\hline $\mathrm{P}$ & 450 & 20,41 & 5,88 & 3,92 & 2,47 & 6,38 & 3,49 & 5,56 & 1,59 & 1,30 \\
\hline $\mathrm{P}$ & 600 & 22,13 & 7,22 & 5,98 & 3,58 & 9,57 & 3,06 & 3,71 & 1,67 & 2,03 \\
\hline $\mathrm{K}$ & 0 & 4,90 & 2,04 & 0,19 & 0,39 & 0,57 & 2,46 & 26,22 & 0,51 & 0,20 \\
\hline K & 50 & 27,30 & 6,86 & 6,11 & 2,99 & 9,09 & 4,00 & 4,53 & 2,05 & 1,52 \\
\hline $\mathrm{K}$ & 100 & 26,83 & 7,15 & 6,01 & 3,26 & 9,27 & 3,77 & 4,45 & 1,85 & 1,67 \\
\hline K & 150 & 25,42 & 7,14 & 6,08 & 3,23 & 9,31 & 3,60 & 4,29 & 1,90 & 1,73 \\
\hline $\mathrm{K}$ & 200 & 28,13 & 7,03 & 6,41 & 3,13 & 9,54 & 4,02 & 4,47 & 2,08 & 1,59 \\
\hline $\mathrm{Ca}$ & 0 & 4,90 & 2,04 & 0,19 & 0,39 & 0,57 & 2,46 & 26,22 & 0,51 & 0,20 \\
\hline $\mathrm{Ca}$ & 0,8 & 28,44 & 7,27 & 6,57 & 3,25 & 9,83 & 3,92 & 4,38 & 2,04 & 1,67 \\
\hline $\mathrm{Ca}$ & 1,0 & 26,83 & 7,15 & 6,01 & 3,26 & 9,27 & 3,77 & 4,45 & 1,85 & 1,67 \\
\hline $\mathrm{Ca}$ & 1,2 & 30,10 & 6,83 & 6,49 & 3,14 & 9,63 & 4,42 & 4,79 & 2,03 & 1,48 \\
\hline $\mathrm{Ca}$ & 1,4 & 28,74 & 6,86 & 6,50 & 3,28 & 9,78 & 4,18 & 4,55 & 1,95 & 1,59 \\
\hline $\mathrm{Mg}$ & 0 & 4,90 & 2,04 & 0,19 & 0,39 & 0,57 & 2,46 & 26,22 & 0,51 & 0,20 \\
\hline Mg & 0,2 & 32,63 & 7,23 & 7,59 & 3,34 & 10,93 & 4,56 & 4,45 & 2,31 & 1,64 \\
\hline $\mathrm{Mg}$ & 0,4 & 26,83 & 7,15 & 6,01 & 3,26 & 9,27 & 3,77 & 4,45 & 1,85 & 1,67 \\
\hline $\mathrm{Mg}$ & 0,6 & 26,58 & 7,30 & 6,39 & 3,49 & 9,88 & 3,66 & 4,26 & 1,84 & 1,82 \\
\hline $\mathrm{Mg}$ & 0,8 & 28,31 & 7,14 & 6,40 & 3,42 & 9,82 & 3,98 & 4,47 & 1,88 & 1,70 \\
\hline S & 0 & 4,90 & 2,04 & 0,19 & 0,39 & 0,57 & 2,46 & 26,22 & 0,51 & 0,20 \\
\hline S & 20 & 28,86 & 7,23 & 6,55 & 3,47 & 10,03 & 4,01 & 4,51 & 1,89 & 1,72 \\
\hline S & 40 & 26,83 & 7,15 & 6,01 & 3,26 & 9,27 & 3,77 & 4,45 & 1,85 & 1,67 \\
\hline S & 60 & 28,32 & 7,35 & 6,74 & 3,61 & 10,35 & 3,88 & 4,31 & 1,87 & 1,83 \\
\hline S & 80 & 28,44 & 7,34 & 6,78 & 3,63 & 10,42 & 3,90 & 4,28 & 1,87 & 1,84 \\
\hline
\end{tabular}

${ }^{1}$ altura (H), diâmetro do coleto (DC), peso de matéria seca da parte aérea (MSPA), peso de matéria seca de raiz (MSR), peso de matéria seca total (MST), altura de parte aérea por diâmetro do coleto (H/D), altura de parte aérea por peso de matéria seca de parte aérea (H/MSPA), peso de matéria seca de parte aérea por peso de matéria seca de raiz (MSPA/MSR) e Índice de Qualidade de Dickson (IQD).

De acordo com Gomes et al. (1990), embora a H das mudas e o DC sejam parâmetros importantes para as análises do padrão de qualidade de mudas, outros autores também recomendam que sejam analisados os pesos de massa seca de parte aérea e de raiz. A produção de massa seca tem sido considerada um dos melhores parâmetros para caracterizar a qualidade de mudas, apresentando, porém, o inconveniente de ser um método destrutivo, o

Cerne, Lavras, v. 18, n. 1, p. 87-98, jan./mar. 2012 
que inviabiliza seu uso na maioria dos viveiros (GOMES, 2001). Cabe ressaltar que a adição de nutrientes ao meio de crescimento possibilitou o incremento em massa seca das mudas de canafístula, independente do nutriente analisado, nos dois compartimentos (parte área e radicular).

De acordo com Gomes (2001), em razão da facilidade de medição, tanto da H quanto do DC, e por ser um método não destrutivo, a relação entre esses parâmetros pode ser considerada e aplicada para muitas das espécies florestais, constituindo-se num dos mais importantes parâmetros morfológicos para estimar o crescimento das mudas após o plantio definitivo no campo. Ainda, de acordo com esse autor, o valor resultante da divisão da altura da parte aérea da muda pelo seu respectivo diâmetro do coleto exprime um equilíbrio de crescimento.

A relação H/MSPA não é, comumente, uma característica usada para avaliar qualidade de mudas, mas pode ser de grande valia, principalmente para predizer o potencial de sobrevivência da muda no campo (GOMES, 2001). De acordo com este autor, quanto menor for esse índice, mais lignificada está a muda e maior a sua capacidade de sobrevivência no campo. Nesse sentido, pode-se observar que houve redução, em pelo menos 6 vezes, nos valores obtidos para mudas cultivadas em substrato não fertilizado versus fertilizado, indicando que a adição de nutrientes promoveu um melhor índice de qualidade de mudas.

A relação entre o peso de massa seca da parte aérea e o do respectivo sistema radicular das mudas é considerada como um quociente eficiente e seguro para expressar o padrão de qualidade destas (GOMES, 2001). Brissette (1984) menciona que em um encontro de pesquisadores ficou estabelecido que um valor igual a 2,0 seria a melhor relação de MSPA/MSR de uma mesma planta. De forma geral, pode-se observar que os valores médios para as menores doses (crescentes) de cada nutriente (Tabela 3) se encontram próximos do valor tido como ideal $(2,0)$, permitindo inferir que, por esse índice e para essas doses, as mudas de canafístula alcançaram um bom padrão de qualidade.

O IQD é uma fórmula balanceada onde estão incluídas as relações das características morfológicas (GOMES, 2001), e que foi desenvolvida, estudando o comportamento de mudas de Picea glauca e Pinus monficola por Dickson et al. (1960). Segundo Gomes (2001), quanto maior o valor desse quociente, melhor o padrão de qualidade das mudas. Assim, a adição de nutrientes ao meio de crescimento, como para outras características, promoveu aumentos nos referidos índices, independente do nutriente avaliado.

\subsection{Efeito do nitrogênio}

Os valores médios observados para as diversas características avaliadas, em geral, diminuíram, em razão da elevação de doses de N (Tabela 3). A aplicação de doses de $\mathrm{N}$ gerou efeitos que resultaram em modelos linear, exceto as características H, H/D e MSPA/MSR (Tabela 4).

Tabela 4 - Estimativas geradas do efeito do $\mathrm{N}$ em $\mathrm{mg} / \mathrm{dm}^{3}$ em mudas de canafístula aos 120 dias após a semeadura, cultivadas em latossolo vermelho amarelo álico.

Table 4 - Estimates of the effect of $\mathrm{N}$ in $\mathrm{mg} / \mathrm{dm}^{3}$ on canafístula seedlings 120 days after planting, using red-yellow alic latosol for cultivation.

\begin{tabular}{lccc}
\hline Característica $^{1}$ & Equação & $\mathrm{R}^{2}$ & $\begin{array}{c}\text { Dose } \\
\text { recomendada }\end{array}$ \\
\hline H & $\hat{Y}=\bar{Y}=27,12$ & & 50 \\
DC & $\hat{Y}=8,125-0,01 * * X$ & 99,72 & 50 \\
MSPA & $\hat{Y}=7,665-0,014 * * * X$ & 90,86 & 50 \\
MSR & $\hat{Y}=3,995-0,006 * * X$ & 78,88 & 50 \\
MST & $\hat{Y}=11,655-0,002 * X$ & 87,90 & 50 \\
H/D & $\hat{Y}=\bar{Y}=3,93$ & & 50 \\
H/MSPA & $\hat{Y}=3,7988+0,007 * X$ & 63,00 & 50 \\
MSPA/MSR & $\hat{Y}=\bar{Y}=1,83$ & & 50 \\
IQD & $\hat{Y}=2,086-0,004 * * X$ & 85,77 & 50 \\
\hline
\end{tabular}

${ }^{1}$ altura (H), diâmetro do coleto (DC), peso de matéria seca da parte aérea (MSPA), peso de matéria seca de raiz (MSR), peso de matéria seca total (MST), altura de parte aérea por diâmetro do coleto (H/D), altura de parte aérea por peso de matéria seca de parte aérea (H/MSPA), peso de matéria seca de parte aérea por peso de matéria seca de raiz (MSPA/MSR) e Índice de Qualidade de Dickson (IQD).

***Significativo a 1\% de probabilidade, **significativo a 5\% de probabilidade e *significativo a $10 \%$ de probabilidade.

$\mathrm{O}$ efeito do $\mathrm{N}$ foi linear positivo para a relação H/MSPA e negativo para DC, MSPA, MSR, MST e IQD, indicando que, de modo geral, maior crescimento e qualidade das mudas de canafístula será obtido com aplicação de doses inferiores a $50 \mathrm{mg} / \mathrm{dm}^{3}$, ou seja, dose entre a dose zero e a menor concentração testada neste estudo. A aplicação de $\mathrm{N}$ resultou em maior crescimento das plantas, no entanto, a espécie apresentou-se menos exigente em $\mathrm{N}$ ou os teores originais de $\mathrm{N}$ no solo fornecido como substrato para a produção das mudas estavam próximos do exigido pela canafístula.

Cerne, Lavras, v. 18, n. 1, p. 87-98, jan./mar. 2012 
Efeitos no crescimento de plantas em função da aplicação de $\mathrm{N}$ são esperados, tendo em vista que o nutriente apresenta papel fundamental no crescimento vegetativo. Efeitos positivos da adubação nitrogenada sobre a produção de diversas espécies florestais arbóreas podem ser encontradas na literatura. Respostas significativas à aplicação de $\mathrm{N}$ foram observadas por Cruz et al. (2006), Gonçalves et al. (2008) e Marques et al. (2006a,b, 2009), os quais observaram efeito positivo do $\mathrm{N}$ sobre o crescimento e qualidade de plantas de Mimosa caesalpinaefolia, Dalbergia nigra, Piptadenia gonoacantha, Samanea inopinata e Anadenathera macrocarpa, respectivamente.

\subsection{Efeito do fósforo}

$\mathrm{O}$ P foi o nutriente mais exigido pelas plantas de canafístula, implicando em aumento dos valores observados para todas as características avaliadas, exceto para a relação H/MSPA (Tabela 3).

Verificou-se efeito linear positivo da adição de $\mathrm{P}$ para o índice IQD e resposta oposta foi observada para a relação H/MSPA (Tabela 5). Houve efeito significativo da aplicação de P para as características DC, MSPA, MSR e MST, sendo o efeito de ordem raiz quadrada (Tabela 5), em que o aumento das doses favoreceu o acréscimo dos valores dessas características até um ponto de máximo, em doses que variaram entre 490,7 a 700,7 mg/dm³ (dose esta superior a maior dose testada), quando então, as médias diminuíram.

As doses recomendadas para canafístula são superiores às encontradas por Gonçalves et al. (2008) para Anadenanthera macrocarpa, sendo que o melhor crescimento das mudas ocorreu com dose de entre 206 a $279 \mathrm{mg} / \mathrm{dm}^{3}$, em condições experimentais semelhantes e utilizando o mesmo tipo de solo (Latossolo Vermelho Amarelo álico), fonte e doses dos fertilizantes fosfatados.

Outros autores também verificaram bom crescimento de mudas cultivadas com dose de $\mathrm{P}$ inferior a recomendada neste estudo. Ceconi et al. (2006) recomendam a aplicação de $360 \mathrm{mg} / \mathrm{dm}^{3}$ de $\mathrm{P}$ na produção de mudas de Luehea divaricata, Gomes et al. (2008) recomendam $54 \mathrm{mg} / \mathrm{dm}^{3}$ de P para Apuleia leiocarpa, Ceconi et al. (2007) recomendam $450 \mathrm{mg} / \mathrm{dm}^{3}$ de P para Ilex paraguariensis. Isso indica que a espécie estudada mostra-se mais exigente em $\mathrm{P}$, o que também foi observado por Lima et al. (2008) e Mello et al. (2008), os quais recomendaram aplicação de doses de $\mathrm{P}$ semelhantes às indicadas para canafístula, na produção de mudas de Acacia mearnsii e Euterpe edulis, respectivamente.

A importância da fertilização fosfatada na produção de mudas de canafístula também foi evidenciada por Venturin et al. (1999), os quais destacaram que a presença de P no meio de crescimento é fundamental

Tabela 5 - Estimativas geradas do efeito do P em mg/dm³ em mudas de canafístula aos 120 dias após a semeadura, cultivadas em latossolo vermelho amarelo álico.

Table 5 - Estimates of the effect of $P$ in $\mathrm{mg} / \mathrm{dm}^{3}$ on canafístula seedlings 120 days after planting, using red-yellow alic latosol for cultivation.

\begin{tabular}{lccc}
\hline Característica $^{1}$ & Equação & $\mathrm{R}^{2}$ & Dose recomendada \\
\hline H & $\hat{Y}=\dot{Y}=22,43$ & & 150 \\
DC & $\hat{Y}=0,849+0,535^{10,31} * * \sqrt{X}-0,012^{15,32} * * X$ & 526,8 \\
MSPA & $\hat{Y}=-5,125+0,997 * \sqrt{X}-0,022^{13,46} * X$ & 58,27 & 490,7 \\
MSR & $\hat{Y}=-0,811+0,322^{15,66} * \sqrt{X}-0,006^{23,97} * X$ & 59,21 & 700,7 \\
MST & $\hat{Y}=-5,936+1,299 * \sqrt{X}-0,028^{15,07} * X$ & 74,98 & 532,9 \\
H/D & $\hat{Y}=\bar{Y}=3,50$ & 61,59 & 150 \\
H/MSPA & $\hat{Y}=6,795-0,005 * * X$ & & 600 \\
MSPA/MSR & $\hat{Y}=\bar{Y}=1,67$ & & 1,35 \\
IQD & $\hat{Y}=0,91+0,002 * * * X$ & & 60,73 \\
\hline
\end{tabular}

${ }^{1}$ altura (H), diâmetro do coleto (DC), peso de matéria seca da parte aérea (MSPA), peso de matéria seca de raiz (MSR), peso de matéria seca total (MST), altura de parte aérea por diâmetro do coleto (H/D), altura de parte aérea por peso de matéria seca de parte aérea (H/MSPA), peso de matéria seca de parte aérea por peso de matéria seca de raiz (MSPA/MSR) e Índice de Qualidade de Dickson (IQD). ***Significativo a $1 \%$ de probabilidade, **significativo a $5 \%$ de probabilidade e *significativo a $10 \%$ de probabilidade.

Cerne, Lavras, v. 18, n. 1, p. 87-98, jan./mar. 2012 
para o crescimento satisfatório da espécie, observando que a mesma é muito exigente em $\mathrm{P}$, e a sua ausência é prejudicial ao adequado crescimento e bom padrão de qualidade das mudas. Outro efeito da aplicação de P com resposta positiva foram verificados por Fernandes et al. (2002, 2007) em mudas de Cordia goeldiana.

\subsection{Efeito do potássio}

A aplicação de doses crescentes de $\mathrm{K}$ provocou poucos efeitos significativos nas diferentes características analisadas (Tabela 6). Para o índice IQD verificou-se efeito quadrático, sendo recomendada a dose de $134,5 \mathrm{mg} / \mathrm{dm}^{3}$ de $\mathrm{K}$, visando à produção de mudas de canafístula com bom padrão de qualidade.

Os resultados obtidos com a aplicação de doses de $\mathrm{K}$ indicam que a necessidade da canafístula por esse nutriente pode ter sido suprida pelos teores de K originais do solo usado como substrato, ou que a espécie apresente pouca exigência nutricional por esse nutriente.

Diversos autores, de maneira semelhante ao observado neste estudo, constataram ausência de efeito da aplicação de K sobre o crescimento e qualidade das mudas. Duboc et al. (1996) também verificaram ausência de efeito da aplicação de K em mudas de Hymenaea courbaril, o mesmo foi observado por Gonçalves et al. (2008) e Souza et al. (2006) em Tabebuia impetiginosa e Anadenathera macrocarpa, respectivamente.

\subsection{Efeito do cálcio}

A espécie não apresentou resposta à aplicação de doses crescentes de Ca (Tabela 3), ou seja, foi observado efeito não significativo para todas as características avaliadas (Tabela 7). Isso indica que a canafístula apresenta baixo requerimento por $\mathrm{Ca}$ ou que os teores originais do nutriente existentes no solo foram suficientes para o adequado crescimento das plantas.

A ausência de efeito da aplicação de Ca sobre o crescimento e qualidade das plantas já foi relatada por outros autores, tais como: Bernardino et al. (2007), Duboc et al. (1996) e Gonçalves et al. (2008) em mudas de Hymenea courbaril, Dalbergia nigra e Anadenathera macrocarpa, respectivamente. No entanto, reposta à aplicação de Ca foi relatada em outras espécies por Cruz et al. (2004), Gomes et al. (2008) e Souza et al. (2008) em mudas de Tabebuia impetiginosa, Apuleia leiocarpa e Machaerium nictitans, respectivamente.

Venturin et al. (1999) observaram resposta contrária à verificada neste estudo com a mesma espécie, ou seja, os autores obtiveram efeito significativo da aplicação de Ca sobre o crescimento das plantas. No entanto, o solo usado como substrato, neste estudo, apresentava teores originais do nutriente superiores ao do solo usado como substrato pelos autores mencionados. Ainda, os autores testaram a elevação dos teores de Ca no solo até $0,49 \mathrm{cmol}_{\mathrm{c}} / \mathrm{dm}^{3}$,

Tabela 6 - Estimativas geradas do efeito do $\mathrm{K}$ em mg/dm³ em mudas de canafístula aos 120 dias após a semeadura, cultivadas em latossolo vermelho amarelo álico.

Table 6 - Estimates of the effect of $\mathrm{K}$ in $\mathrm{mg} / \mathrm{dm}^{3}$ on canafístula seedlings 120 days after planting, using red-yellow alic latosol for cultivation.

\begin{tabular}{|c|c|c|c|}
\hline Característica $^{1}$ & Equação & $\mathrm{R}^{2}$ & Dose recomendada \\
\hline $\mathrm{H}$ & $\hat{Y}=\bar{Y}=26,92$ & & 50 \\
\hline DC & $\hat{Y}=\bar{Y}=7,05$ & & 50 \\
\hline MSPA & $\hat{Y}=\bar{Y}=6,15$ & & 50 \\
\hline MSR & $\hat{Y}=\bar{Y}=3,15$ & & 50 \\
\hline MST & $\hat{Y}=\bar{Y}=9,30$ & & 50 \\
\hline $\mathrm{H} / \mathrm{D}$ & $\hat{Y}=\bar{Y}=3,85$ & & 50 \\
\hline H/MSPA & $\hat{Y}=\bar{Y}=4,44$ & & 50 \\
\hline MSPA/MSR & $\hat{Y}=\bar{Y}=1,97$ & & 50 \\
\hline IQD & $\hat{Y}=-0,00002 * X^{2}+0,015 * X+0,855$ & 69,92 & 134,5 \\
\hline
\end{tabular}

1altura (H), diâmetro do coleto (DC), peso de matéria seca da parte aérea (MSPA), peso de matéria seca de raiz (MSR), peso de matéria seca total (MST), altura de parte aérea por diâmetro do coleto (H/D), altura de parte aérea por peso de matéria seca de parte aérea (H/MSPA), peso de matéria seca de parte aérea por peso de matéria seca de raiz (MSPA/MSR) e Índice de Qualidade de Dickson (IQD). ***Significativo a $1 \%$ de probabilidade, ${ }^{* *}$ significativo a $5 \%$ de probabilidade e *significativo a $10 \%$ de probabilidade.

Cerne, Lavras, v. 18, n. 1, p. 87-98, jan./mar. 2012 
Tabela 7 - Estimativas geradas do efeito do Ca em $\mathrm{cmol}_{\mathrm{c}} / \mathrm{dm}^{3} \mathrm{em}$ mudas de canafístula aos 120 dias após a semeadura, cultivadas em latossolo vermelho amarelo álico.

Table 7 - Estimates of the effect of Ca in cmolc/ $\mathrm{dm}^{3}$ on canafístula seedlings 120 days after planting, using red-yellow alic latosol for cultivation.

\begin{tabular}{lcc}
\hline Característica $^{1}$ & \multicolumn{1}{c}{ Equação } & Dose recomendada \\
\hline H & $\hat{Y}=\bar{Y}=28,53$ & 0,8 \\
DC & $\hat{Y}=\bar{Y}=7,03$ & 0,8 \\
MSPA & $\hat{Y}=\bar{Y}=6,39$ & 0,8 \\
MSR & $\hat{Y}=\bar{Y}=3,23$ & 0,8 \\
MST & $\hat{Y}=\bar{Y}=9,63$ & 0,8 \\
H/D & $\hat{Y}=\bar{Y}=4,07$ & 0,8 \\
H/MSPA & $\hat{Y}=\bar{Y}=4,54$ & 0,8 \\
MSPA/MSR & $\hat{Y}=\bar{Y}=1,97$ & 0,8 \\
IQD & $\hat{Y}=\bar{Y}=1,60$ & 0,8 \\
\hline
\end{tabular}

${ }^{1}$ altura (H), diâmetro do coleto (DC), peso de matéria seca da parte aérea (MSPA), peso de matéria seca de raiz (MSR), peso de matéria seca total (MST), altura de parte aérea por diâmetro do coleto (H/D), altura de parte aérea por peso de matéria seca de parte aérea (H/MSPA), peso de matéria seca de parte aérea por peso de matéria seca de raiz (MSPA/MSR) e Índice de Qualidade de Dickson (IQD).

***Significativo a $1 \%$ de probabilidade, **significativo a $5 \%$ de probabilidade e *significativo a $10 \%$ de probabilidade.

sendo esta dosagem inferior a menor dose testada no experimento em questão, o que pode explicar a diferença de resultados encontrada.

De acordo com Neves et al. (1982), a calagem, como forma de adicionar cálcio ao meio de crescimento, somente seria necessária quando o nível do mesmo for inferior a $0,2 \mathrm{cmol} / \mathrm{dm}^{3}$, o que corrobora com os resultados constatados neste experimento, tendo em vista que o solo usado como substrato apresentava exatamente esta concentração, ou seja, $0,2 \mathrm{cmol}_{\text {c }} / \mathrm{dm}^{3}$ (Tabela 1 ).

\subsection{Efeito do magnésio}

O efeito da aplicação de Mg sobre as características avaliadas só foi significativo para a relação MSPA/MSR (Tabela 8), sendo esse feito linear negativo, ou seja, as doses crescentes de $\mathrm{Mg}$ proporcionaram queda nos valores dessa relação. Portanto, maior qualidade das mudas será obtida com doses inferiores a $0,2 \mathrm{cmol} / \mathrm{cm}^{3}$.

$\mathrm{O}$ fato de poucos efeitos significativos terem sido observados indicam que a espécie tem baixa exigência
Tabela 8 - Estimativas geradas do efeito do $\mathrm{Mg} \mathrm{em} \mathrm{cmol}_{c} / \mathrm{dm}^{3} \mathrm{em}$ mudas de canafístula aos 120 dias após a semeadura, cultivadas em latossolo vermelho amarelo álico.

Table 8 - Estimates of the effect of $\mathrm{Mg}$ in $\mathrm{cmolc} / \mathrm{dm}^{3}$ on canafístula seedlings 120 days after planting, using red-yellow alic latosol for cultivation.

\begin{tabular}{|c|c|c|c|}
\hline Característica $^{1}$ & Equação & $\mathrm{R}^{2}$ & $\begin{array}{c}\text { Dose } \\
\text { recomendada }\end{array}$ \\
\hline $\mathrm{H}$ & $\hat{Y}=\bar{Y}=28,58$ & & 0,2 \\
\hline DC & $\hat{Y}=\bar{Y}=7,20$ & & 0,2 \\
\hline MSPA & $\hat{Y}=\bar{Y}=6,60$ & & 0,2 \\
\hline MSR & $\hat{Y}=\bar{Y}=3,38$ & & 0,2 \\
\hline MST & $\hat{Y}=\bar{Y}=9,98$ & & 0,2 \\
\hline $\mathrm{H} / \mathrm{D}$ & $\hat{Y}=\bar{Y}=3,99$ & & 0,2 \\
\hline H/MSPA & $\hat{Y}=\bar{Y}=4,41$ & & 0,2 \\
\hline MSPA/MSR & $\hat{Y}=2,262-0,5569 * X$ & 43,36 & 0,2 \\
\hline IQD & $\hat{Y}=\bar{Y}=1,70$ & & 0,2 \\
\hline
\end{tabular}

${ }^{1}$ altura (H), diâmetro do coleto (DC), peso de matéria seca da parte aérea (MSPA), peso de matéria seca de raiz (MSR), peso de matéria seca total (MST), altura de parte aérea por diâmetro do coleto (H/D), altura de parte aérea por peso de matéria seca de parte aérea (H/MSPA), peso de matéria seca de parte aérea por peso de matéria seca de raiz (MSPA/MSR) e Índice de Qualidade de Dickson (IQD).

***Significativo a $1 \%$ de probabilidade, ${ }^{* *}$ significativo a $5 \%$ de probabilidade e *significativo a $10 \%$ de probabilidade.

por Mg. Esse comportamento também foi observado por Bernardino et al. (2007), Duboc et al. (1996) e Gonçalves et al. (2008) em mudas de Hymenaea courbaril, Dalbergia nigra e Anadenathera macrocarpa, respectivamente. Em contrapartida, Cruz et al. (2004), Gomes et al. (2008) e Souza et al. (2008) encontraram efeito positivo da aplicação de $\mathrm{Mg}$ sobre o crescimento das mudas de Tabebuia impetiginosa, Apuleia leiocarpa e Machaerium nictitans, respectivamente.

Venturin et al. (1999) relatam que a canafístula respondeu à aplicação de Mg, sendo essa reposta contrária à observada neste estudo. No entanto, cabe ressaltar que a dose testada por esses autores foi semelhante à menor dose testada neste experimento. Como na análise de contrastes ortogonais verificou-se efeito significativo da aplicação de macronutrientes ao solo usado como substrato, podese afirmar que a dose recomendada para a produção de mudas de canafístula está entre o teor original de Mg no solo, ou seja, 0 (zero) $\mathrm{cmol}_{\mathrm{c}} / \mathrm{dm}^{3}$, e a menor dose testada: $0,2 \mathrm{cmol}_{\mathrm{c}} / \mathrm{dm}^{3}$.

Cerne, Lavras, v. 18, n. 1, p. 87-98, jan./mar. 2012 


\subsection{Efeito do enxofre}

O crescimento das plantas sob condições diferenciadas de $\mathrm{S}$ só se mostrou diferente significativamente para a característica MSR (Tabela 9). O efeito observado para MSR foi linear positivo, ou seja, maior crescimento radicular será obtido com doses superiores a $80 \mathrm{mg} / \mathrm{dm}^{3}$.

Tabela 9 - Estimativas geradas do efeito do $\mathrm{S}$ em $\mathrm{mg} / \mathrm{dm}^{3}$ em mudas de canafístula aos 120 dias após a semeadura, cultivadas em latossolo vermelho amarelo álico.

Table 9 - Estimates of the effect of $S$ in $\mathrm{mg} / \mathrm{dm} 3$ on canafístula seedlings 120 days after planting, using red-yellow alic latosol for cultivation.

\begin{tabular}{|c|c|c|c|}
\hline Característica $^{1}$ & Equação & $\mathrm{R}^{2}$ & $\begin{array}{c}\text { Dose } \\
\text { recomendada }\end{array}$ \\
\hline $\mathrm{H}$ & $\hat{Y}=\bar{Y}=28,11$ & & 20 \\
\hline DC & $\hat{Y}=\bar{Y}=7,27$ & & 20 \\
\hline MSPA & $\hat{Y}=\bar{Y}=6,52$ & & 20 \\
\hline MSR & $\hat{Y}=3,37+0,006 * X$ & 20,64 & 80 \\
\hline MST & $\hat{Y}=\bar{Y}=10,02$ & & 20 \\
\hline $\mathrm{H} / \mathrm{D}$ & $\hat{Y}=\bar{Y}=3,89$ & & 20 \\
\hline H/MSPA & $\hat{Y}=\bar{Y}=4,39$ & & 20 \\
\hline MSPA/MSR & $\hat{Y}=\bar{Y}=1,87$ & & 20 \\
\hline IQD & $\hat{Y}=\bar{Y}=1,77$ & & 20 \\
\hline
\end{tabular}

${ }^{1}$ altura (H), diâmetro do coleto (DC), peso de matéria seca da parte aérea (MSPA), peso de matéria seca de raiz (MSR), peso de matéria seca total (MST), altura de parte aérea por diâmetro do coleto (H/D), altura de parte aérea por peso de matéria seca de parte aérea (H/MSPA), peso de matéria seca de parte aérea por peso de matéria seca de raiz (MSPA/MSR) e Índice de Qualidade de Dickson (IQD).

***Significativo a $1 \%$ de probabilidade, ${ }^{* *}$ significativo a $5 \%$ de probabilidade e *significativo a $10 \%$ de probabilidade.

A falta de resposta à aplicação de enxofre já foi observada por Dias et al. (1992), Duboc et al. (1996) e Mendonça et al. (1999) em mudas de Sclerolobium paniculatum, Hymenaea courbaril e Myracrodruon urundeuva, respectivamente. No entanto, Balieiro et al. (2001), observaram para Acacia holosericea resposta positiva à aplicação de $\mathrm{S}$.

Segundo Alvarez et al. (1987), respostas a S não têm sido observadas quando as plantas recebem doses insuficientes de fósforo. Como, neste estudo, a espécie mostrou-se muito exigente em $\mathrm{P}$, e nos tratamentos com doses de $\mathrm{S}$ foram aplicadas doses de $\mathrm{P}$ inferiores às exigidas pela planta, pode-se alegar que a ausência de resposta ao $S$ observada neste estudo pode estar relacionada à baixa disponibilidade de $\mathrm{P}$.

\section{CONCLUSÕES}

As mudas de canafístula respondem à adição de macronutrientes, sendo a fertilização importante para o adequado crescimento e qualidade de mudas da espécie.

Com base nos dados de matéria seca total, característica que melhor reflete a produção, as doses de macronutrientes recomendadas para a produção de mudas de canafístula são: $50 \mathrm{mg} / \mathrm{dm}^{3}$ de N; $540 \mathrm{mg} / \mathrm{dm}^{3}$ de P; 50 $\mathrm{mg} / \mathrm{dm}^{3} \mathrm{de} \mathrm{K}$; 0,8 $\mathrm{cmol}_{\mathrm{c}} / \mathrm{dm}^{3}$ de Ca; 0,2 $\mathrm{cmol}_{\mathrm{c}} / \mathrm{dm}^{3} \mathrm{de} \mathrm{Mg}$ e $20 \mathrm{mg} / \mathrm{dm}^{3}$ de $\mathrm{S}$, em Latossolo Vermelho Amarelo álico como substrato.

As mudas de canafístula mostraram-se bastante exigentes em P e menos exigente em N, K, Ca, Mg e S.

São necessários estudos adicionais com os nutrientes $\mathrm{N}, \mathrm{K}, \mathrm{Ca}, \mathrm{Mg}$ e $\mathrm{S}$, com doses inferiores às menores doses testadas, tendo em vista que, segundo dados deste estudo, a melhor dose desses nutrientes encontra-se entre os teores originais do solo e a menor dose aplicada.

\section{REFERÊNCIAS}

ALVAREZ, V. H. Equilíbrio de formas disponíveis de fósforo e enxofre em dois Latossolos de Minas Gerais. 1974. 125 f. Dissertação (Mestrado em Solos e Nutrição de Plantas) - Universidade Federal de Viçosa, Viçosa, 1974.

ALVAREZ, V. H.; FREIRE, F. M.; GUIMARÃES, P. T. G. Concentrações relativas ótimas de nitrogênio, fósforo e enxofre, na adubação do cafeeiro, num latossolo vermelho escuro de Machado, MG. Pesquisa Agropecuária Brasileira, Brasília, v. 22, n. 2, p. 145-152, fev. 1987.

BALIEIRO, F. C.; OLIVEIRA, I. G.; DIAS, L. E. Formação de mudas de Acacia holosericea e Acacia auriculiformis: resposta à calagem, fósforo, potássio e enxofre. Revista Árvore, Viçosa, v. 25, n. 2, p. 183-191, mar./abr. 2001.

BERNARDINO, D. C. de S.; PAIVA, H. N. de; NEVES, J. C. L.; GOMES, J. M.; MARQUES, V. B. Influência da saturação por bases e da relação Ca:Mg do substrato sobre o crescimento inicial de jacarandá-da-bahia (Dalbergia nigra (Vell.) Fr. All. Ex Benth.). Revista Árvore, Viçosa, v. 31, n. 4, p. 567-573, jul./ago. 2007.

Cerne, Lavras, v. 18, n. 1, p. 87-98, jan./mar. 2012 
CARNEIRO, J. G. A. Produção e controle de qualidade de mudas florestais. Curitiba: UFPR/FUPEF, 1995. 451 p.

CARVALHO, M. M. Ações visando recuperação de áreas de pastagens degradadas. In: ENCONTRO PARA CONSERVAÇÃO DA NATUREZA, 1., 1997, Viçosa, MG. Anais... Viçosa, MG: CMCN/DEF/UFV, 1997. p. 202-206.

CARVALHO, P. E. R. Espécies arbóreas brasileiras.

Colombo: Embrapa Florestas, 2003. v. 1, 1039 p.

CECONI, D. E.; POLETTO, I.; BRUN, E. J.; LOVATO, T. Crescimento de mudas de açoita-calavo (Luehea divaricata Mart.) sob influência da adubação fosfatada. Cerne, Lavras, v. 12, n. 3, p. 292-299, jul./set. 2006.

CECONI, D. E.; POLLETO, I.; LOVATO, T.; MUNIZ, M. F. B. Exigência nutricional de mudas de erva-mate (Ilex paraguariensis A. St.-Hil.) à adubação fosfatada. Ciência Florestal, Santa Maria, v. 17, n. 1, p. 25-32, 2007.

CRUZ, C. A. F.; PAIVA, H. N.; GOMES, K. C. O.; GUERRERO, C. R. A. Efeito de diferentes níveis de saturação por bases no desenvolvimento e qualidade de mudas de ipê-roxo (Tabebuia impetiginosa (Mart.) Standley). Scientia Forestalis, Piracicaba, n. 66, p. 100-107, 2004.

CRUZ, C. A. F.; PAIVA, H. N.; GUERRERO, C. R. A. Efeito da adubação nitrogenada na produção de mudas de setecascas (Samanea inopinata (Harms) Ducke). Revista Árvore, Viçosa, v. 30, n. 4, p. 537-546, jul./ago. 2006.

DIAS, L. E.; JUCKSCH, I.; ALVAREZ, V. H.; BARROS, N. F.; BRIENZA JÚNIOR, S. Formação de mudas de táxi-branco (Sclerolobium paniculatum Voguel): II., resposta a nitrogênio, potássio e enxofre. Revista Árvore, Viçosa, v. 16, n. 2, p. 135-143, 1992.

DICKSON, A.; LEAF, A. L.; HOSNER, J. F. Quality appraisal of white spruce and white pine seedling stock in nurseries.

Forestry Chronicle, v. 36, p. 10-13, 1960.

DUBOC, E.; VENTURIN, N.; VALE, F. R. do; DAVIDE, A. C. Nutrição do jatobá (Hymenaea courbaril L. var. Stilbocarpa (Hayne) Lee et Lang.). Cerne, Lavras, v. 2, n. 1, p. 31-47, jan./jun. 1996.

FERNANDES, A. R.; CARVALHO, J. G.; PAIVA, H. N.; MIRANDA, J. R. P. Efeito do fósforo e do zinco sobre o crescimento de mudas de freijó (Cordia goeldiana Huber.). Revista de Ciências Agrárias, Belém, n. 37, p. 123-131, 2002.

FERNANDES, A. R.; PAIVA, H. N. de; CARVALHO, J. G.; MIRANDA, J. R. P. Crescimento e absorção de nutrientes por mudas de freijó (Cordia goeldiana Huber) em função de doses de fósforo e zinco. Revista Árvore, Viçosa, v. 31, n. 4, p. 599608, jul./ago. 2007.

GOMES, J. M. Parâmetros morfológicos na avaliação da qualidade de mudas de Eucalyptus grandis, produzidas em diferentes tamanhos de tubete e de dosagens de N-P-K. 2001. 126 p. Tese (Doutorado em Ciência Florestal) Universidade Federal de Viçosa, Viçosa, 2001.

GOMES, J. M.; COUTO, L.; BORGES, R. C. G.; FREITAS, S. C. Influência do tamanho da embalagem plástica na produção de mudas de ipê (Tabebuia serratifolia) de copaíba (Copaifera langsdorffii) e de angico vermelho (Piptadenia peregrina). Revista Árvore, Viçosa, v. 14, n. 1, p. 26-34, 1990.

GOMES, K. C. de O.; PAIVA, H. N. de; NEVES, J. C. L.; BARROS, N. F.; SILVA, S. R. Crescimento de mudas de garapa em reposta à calagem e ao fósforo. Revista Árvore, Viçosa, v. 32, n. 3, p. 387-394, maio/jun. 2008.

GONÇALVES, E. de O.; PAIVA, H. N. de; NEVES, J. C. L.; GOMES, J. M. Crescimento de mudas de angico-vermelho (Anadenanthera macrocarpa (Benth.) Brenan) sob diferentes doses de macronutrientes. Revista Árvore, Viçosa, v. 32, n. 6, p. 1029-1040, nov./dez. 2008.

GONÇALVES, J. L. M.; KAGEYAMA, P. Y.; FREIXEDAS, V. M.; GONÇALVES, J. C.; GERES, W. L. A. Capacidade de absorção e eficiência nutricional de algumas espécies arbóreas tropicais. Revista do Instituto Florestal, Curitiba, v. 4, n. 2, p. 463-469, 1992.

LIMA, L. S. H.; FRANCO, E. T.; SCHUMACHER, M. V. Crescimento de mudas de Euterpe edulis Martius em reposta a diferentes doses de fósforo. Ciência Florestal, Santa Maria, v. 18, n. 4, p. 461-470, 2008.

LORENZI, H. Árvores brasileiras: manual de identificação e cultivo de plantas arbóreas nativas do Brasil. Nova Odessa: Plantarum, 1992. 352 p.

Cerne, Lavras, v. 18, n. 1, p. 87-98, jan./mar. 2012 
MARQUES, L. S.; PAIVA, H. N. de; NEVES, J. C. L.; GOMES, J. M.; SOUZA, P. H. de. Crescimento de mudas de jacaré (Piptadenia gonoacantha J. F. Macbr.) em diferentes tipos de solos e fontes e doses de nitrogênio. Revista Árvore, Viçosa, v. 33, n. 1, p. 81-91, jan./fev. 2009.

MARQUES, V. B.; PAIVA, H. N. de; GOMES, J. M.; NEVES, J. C. L. Efeito de fontes e doses de nitrogênio no crescimento de mudas de sabiá (Mimosa caesalpiniaefolia Benth.).

Scientia Forestalis, Piracicaba, n. 71, p. 77-85, 2006a.

MARQUES, V. B.; PAIVA, H. N. de; GOMES, J. M.; NEVES, J. C. L.; BERNARDINO, D. C. de S. Efeito de fontes e doses de nitrogênio sobre o crescimento de inicial e qualidade de mudas de jacarandá-da-bahia (Dalbergia nigra (Vell.) Fr. All. Ex Benth.). Revista Árvore, Viçosa, v. 30, n. 5, p. 725-735, set./out. 2006b.

MELLO, A. H. de; KAMINSKI, J.; ANTONIOLLI, Z. I.; SANTOS, L. C. dos; SOUZA, E.; LORENSI, D.; SCHIRMER, G. K.; GOULART, R. M. Influência de substratos e fósforo na produção de mudas micorrizadas de Acacia mearnsii de Wild. Ciência Florestal, Santa Maria, v. 18, n. 3, p. 321-327, maio/jun. 2008.

MENDONÇA, A. V. R.; NOGUEIRA, F. D.; VENTURIN, N.; SOUZA, J. S. Exigências nutricionais de Myracroduon urundeuva Fr. All (Aroeira do Sertão). Cerne, Lavras, v. 5, n. 2, p. 65-75, jul./dez. 1999.

NEVES, J. C. L.; BARROS, N. F.; NOVAIS, R. F.; ANJOS, J. L. Efeito do alumínio em amostras de dois latossolos sob cerrado sobre o crescimento e absorção de nutrientes de mudas de Eucalyptus spp. Revista Árvore, Viçosa, v. 6, n. 1, p. 17-28, 1982.

SOUZA, P. A. de; VENTURIN, N.; MACEDO, R. L. G. de. Adubação mineral do ipê-roxo (Tabebuia impetiginosa). Ciência Florestal, Santa Maria, v. 16, n. 3, p. 261-270, maio/jun. 2006.

SOUZA, P. H. de; PAIVA, H. N. de; NEVES, J. C. L.; GOMES, J. M.; MARQUES, L. S. Influência da saturação por bases do substrato no crescimento e qualidade de mudas de Machaerium nictitans (Vell.) Benth. Revista Árvore, Viçosa, v. 32, n. 2, p. 193-201, mar./abr. 2008.

TURRENT, F. A. Uso de una matriz mixta para la optimización de cinco a ocho factores controlables de la producción. Chapingo: Rama de Suelos, 1979. 65 p. (Boletim técnico, 6).

VENTURIN, N.; DUBOC, E.; VALE, F. R. do; DAVIDE, A. C. Adubação mineral do Angico-Amarelo (Peltophorum dubium (SPRENG.) TAUB.). Pesquisa Agropecuária Brasileira, Brasília, v. 34, n. 3, p. 441-448, mar. 1999.

Cerne, Lavras, v. 18, n. 1, p. 87-98, jan./mar. 2012 